\title{
Planes de cuidados enfermeros de estudiantes de pregrado: comparación de dos modelos
}

\section{Nursing care plans of undergraduate students: Comparison of two models}

\author{
Luz-Eugenia Ibáñez-Alfonso ${ }^{1}$; María-Teresa Fajardo-Peña'; Claudia-Esperanza Cardozo-Ortiz ${ }^{\text {; }}$ Zayne-Milena Roa-Díaz ${ }^{1}$
}

Forma de citar: Ibañez-Alfonso LE, Fajardo-Peña MT, Cardozo-Ortiz CE, Roa-Díaz ZM. Planes de cuidados enfermeros de estudiantes de pregrado: comparación de dos modelos. Salud UIS. 2020; 52(1): 33-40. doi: http://dx.doi.org/10.18273/revsal.v52n1-2020005 (c) (i)

\section{Resumen}

Introducción: El modelo AREA (Análisis de Resultado Esperado Actual) plantea la importancia de reorientar la enseñanza y el aprendizaje del proceso de enfermería al pensarlo en términos más amplios, panorámicos y reflexivos; facilitando el desarrollo de habilidades de razonamiento clínico, lo que constituye un gran avance para la enseñanza y la práctica en la toma de decisiones clínicas que integra NANDA, NIC y NOC. Los nuevos lineamientos estructurados desde el modelo OPT superan la visión lineal, fraccionada e instrumental de las cinco etapas del proceso de enfermería. Objetivo: Comparar dos modelos de aplicación del proceso de enfermería (generación 2 y 3), en la elaboración de planes de cuidados por parte de estudiantes del programa de enfermería de una institución de educación superior de Bucaramanga (Colombia). Metodología: Estudio de corte transversal. Participaron 33 estudiantes de quinto nivel del programa de enfermería de la UIS. Se definieron las variables y se diseñaron los instrumentos que permitieron evaluar y comparar la aplicación del proceso de enfermería de la segunda y tercera generación. Resultados: Se identificó preferencia por el modelo tercera generación como guía para el abordaje de casos clínicos; sin embargo, es importante resaltar que los estudiantes no encontraron diferencias significativas en aspectos de articulación teórica, ni en la aplicación de los lenguajes NIC y NOC. Los estudiantes encuentran diferencias significativas en el uso del modelo OPT en aspectos relacionados con el razonamiento clínico, la determinación de la esencia del caso, el respaldo diagnóstico y la identificación del diagnóstico de enfermería. Conclusiones: Ampliar la enseñanza del modelo OPT en todos los cursos del programa de enfermería UIS, facilitará una mayor comprensión de los procesos de pensamiento del estudiante y mayor capacidad de adaptar experiencias para satisfacer las necesidades individuales de aprendizaje; igualmente, fortalecerá el currículo y por ende las prácticas docentes.

Palabras clave: Proceso de enfermería; Diagnóstico de Enfermería; Estudiante; Modelo educativo; Planificación de la atención del paciente.

\begin{abstract}
Introduction: The OPT model (Outcome Present State Test) raises the importance of reorienting the teaching and learning of the nursing process when thinking about it in broader, panoramic and reflective terms; it facilitates the development of clinical reasoning skills, which is a breakthrough for teaching and practice in making clinical
\end{abstract}

1. Universidad Industrial de Santander. Bucaramanga, Colombia.

Correspondencia: María Teresa Fajardo. Carrera 32. 29-31 Bucaramanga. Teléfono: +57 76345745 Correo electrónico: mfajardo@uis.edu.co 
decisions that integrate NANDA, NIC and NOC. The new guidelines structured from the OPT model, overcome the linear, fractional and instrumental vision of the five stages of the nursing process. Objective: To compare two nursing process application models (generation 2 and generation 3), in the elaboration of care plans by students of the nursing program of a higher education institution of Bucaramanga (Colombia). Methodology: Cross-sectional study. With participation of 33 students from the fifth level of UIS nursing program UIS. Variables were defined and the instruments to evaluate and compare the application of the nursing process of the second and third generations were designed. Results: Preference for the third generation model was identified as a guide for addressing clinical cases; however, it is important to note that the students did not find significant differences in aspects of theoretical articulation, nor in the application of NIC and NOC languages. Students find significant differences in the use of OPT model in aspects related to clinical reasoning, case essence determination, diagnostic support and in the nursing diagnosis identification. Conclusions: Expanding the teaching of the OPT model in all courses of the nursing program UIS, will facilitate a greater understanding of the student's thinking processes and a greater ability to adapt experiences to meet individual learning needs; it will also strengthen the curriculum and therefore the teaching practices.

Keywords: Nursing process; Nursing diagnosis; Student; Educational model; Patient care planning.

\section{Introducción}

En la actualidad el modelo Outcome Present State Test -OPT (en español modelo AREA: Análisis de Resultado Esperado Actual), es una herramienta que establece una estructura para el razonamiento clínico de los problemas, centrándolos en los resultados esperados en la práctica enfermera. Plantea la importancia de reorientar la enseñanza y el aprendizaje del proceso de enfermería al pensarlo en términos más amplios, panorámicos y reflexivos; facilitando el desarrollo de habilidades de razonamiento clínico, y es por esto que se ha extendido su aplicación a nivel mundial. Para Pesut y Herman, su modelo fue descrito como un modelo de proceso de enfermería tercera generación ${ }^{1}$, definiendo el razonamiento clínico como el pensamiento reflexivo, concurrente, creativo y crítico, inmerso en la práctica de enfermería. El modelo $\mathrm{OPT}^{2}$ requiere que se utilicen todos los elementos del proceso de enfermería para construir y perfeccionar aún más las habilidades de pensamiento de enfermería.

Con el surgimiento de teorías en enfermería en Estados Unidos, que plantearon una herramienta metodológica como es el proceso de enfermería se ha venido extendiendo a nivel mundial, desde 1973, incorporando también la utilización de los lenguajes estandarizados internacionales como son: Clasificación de Diagnósticos de Enfermería (NANDA-I) ${ }^{3}$, Clasificación de Intervenciones de Enfermería (NIC) ${ }^{4}$ y Clasificación de Resultados de Enfermería (NOC) ${ }^{5}$, estos lenguajes permiten que la enfermería denomine de manera estandarizada las respuestas humanas que se ven alteradas en procesos de salud-enfermedad, que denomine unificadamente las intervenciones que realiza en su ejercicio profesional y finalmente que pueda mediante resultados e indicadores también estandarizados, medir la efectividad de sus planes de cuidado, antes y después de las intervenciones realizadas.

Los estudios realizados en diferentes países han demostrado nuevos aportes que es necesario incorporar en los procesos de enseñanza y aprendizaje, al realizar críticas conceptuales al proceso de enfermería tradicional y analizar el uso del modelo OPT como facilitador del aprendizaje autorregulado, reflexivo y articulador; que proporciona una estrategia a educadores y estudiantes en el proceso educativo en diferentes contextos. Lo anterior, fortalece no solo el conocimiento mediante el uso de estrategias de pensamiento crítico para la comprensión de diagnósticos, resultados e intervenciones de enfermería, sino también, el conocimiento metacognitivo mediante la reflexión y la autorregulación, para controlar los procesos cognitivos, identificando beneficios y aplicaciones pedagógicas ${ }^{6-8}$.

Según Pink ${ }^{9}$, el modelo OPT abarca diferentes tipos de pensamiento necesarios para la emergente era conceptual o de pensamiento panorámico. Da gran importancia a la historia y a la narración del paciente, utiliza la empatía para forjar relaciones a través de los cuidados y síntesis de los elementos en un todo; lo que constituye un gran avance para la enseñanza y la práctica en la toma de decisiones clínicas que integra NANDA-I, NIC, NOC ${ }^{5}$. Además, proporciona una estructura idónea para el pensamiento centrado en resultados, lo que potencia la mejora de la calidad al optimizar la evaluación de la efectividad, en lugar de centrarse solo en los problemas desde el enfoque tradicional, lineal y escalonado. 
El modelo OPT plantea la importancia de analizar el proceso de razonamiento clínico, el cual debe considerar simultáneamente los problemas y los resultados mediante la yuxtaposición de ambos y requiere relaciones simultáneas entre NANDA-I, NIC, NOC, atendiendo a las evidencias que se utilizarán para realizar juicios del pensamiento enfermero que permitan tomar decisiones pertinentes a las necesidades del sujeto de cuidado9.

Un currículo que implemente este modelo requiere pensar en aspectos pedagógicos que permitan introducir nuevas formas de enseñanza, aprendizaje y la aplicación práctica de los cuidados. Si bien, las etapas del proceso: valoración, diagnóstico, planeación, ejecución y evaluación, se mantienen; el modelo presenta una nueva perspectiva que requiere el desarrollo de procesos mentales globales, enfocados en la narrativa del sujeto de cuidado; de hecho, la valoración de enfermería se realiza a través de una escucha activa, empática y compasiva ${ }^{1}$.

Pesut y Herman ${ }^{1}$, en su modelo proponen una serie de conceptos agrupados en una hoja de trabajo, como son: persona en la historia, red de razonamiento clínico, lógica clave, problema clave, estado actual, estado de resultado, prueba, contextualización, toma de decisiones, juicio y salida. La persona en la historia hace referencia al problema inicial o al diagnóstico médico, datos de la historia clínica, signos y síntomas, datos de laboratorio, información familiar y/o social entre otros.

La red de razonamiento clínico es una representación gráfica, que permite identificar el problema clave, examinando las relaciones entre los diagnósticos NANDA- $\mathrm{I}^{10}$, facilitando la priorización del diagnóstico central. Equivale a utilizar una lente a través de la cual se ve e interpreta la historia del paciente ${ }^{1}$. El encuadre de la historia del paciente facilita la aplicación de un modelo, una teoría, una perspectiva evolutiva o un conjunto de políticas y procedimientos, para que la enfermera piense como enfermera en lugar de pensar desde una perspectiva ajena como la médica, psicológica o sociológica ${ }^{8,11}$.

En el modelo se explica la importancia de incorporar la lógica clave (cue logic), la cual se refiere a todos los diagnósticos de enfermería NANDA-I propuestos en la red de razonamiento clínico y que conecta con la siguiente etapa del modelo llamada el problema clave (keystone). En esta etapa, se reporta el diagnóstico de enfermería NANDA-I que se ha identificado como el problema principal en la red de razonamiento clínico, dado que tiene el mayor número de conexiones llegando o saliendo.

El estado actual representa una descripción del paciente en la situación inicial que se irá modificando como consecuencia de los cuidados de enfermería. Éste se expresa en términos de los diagnósticos de enfermería NANDA-I, con sus características definitorias, factores de riesgo o factores relacionados, que aportan un marco estructural y dotan de significado a las etapas anteriores. Pesut y Herman $^{1}$, resaltan la forma como la enfermera crea una red de razonamiento clínico para priorizar el estado actual tras haber identificado las relaciones entre todos los diagnósticos NANDA-I.

El estado de resultado incluye los resultados $\mathrm{NOC}^{5}$, que representan una conducta, conducta o percepción que se mide a lo largo de un continuo en respuesta a una intervención de enfermería. Cada resultado ofrece un grupo de indicadores que en el modelo se plantea como medición y se utiliza para determinar la condición del paciente en relación con ese resultado; estos deben ser coherentes con el diagnóstico de enfermería. De esta manera, el proceso de pensamiento que pretende averiguar la forma en que se irán llenando los vacíos existentes entre el estado actual y el resultado esperado; requiere un análisis que yuxtapone el estado actual con los resultados y se tienen en cuenta las intervenciones NIC que se pueden usar para salvar ese vacío ${ }^{1}$.

La toma de decisiones, es el proceso en el que se seleccionan e implementan las intervenciones de enfermería específicas, para lograr los resultados esperados. Existen seis factores que facilitan la selección de las intervenciones: resultados deseados por el paciente, características de los diagnósticos de enfermería, base de investigación para la intervención, viabilidad para la implementación de la intervención, aceptabilidad de la intervención por el paciente y habilidad de la enfermera ${ }^{4}$.

El Juicio es el momento de sacar conclusiones basadas en las acciones realizadas, dando lugar a la reflexión sobre el grado de concordancia entre el estado actual del paciente y los resultados, éstos ayudarán a la autocorrección, auto refuerzo y autoevaluación del propio pensamiento sobre la tarea o situación desde la perspectiva propia de la profesión ${ }^{1}$; lo cual a su vez permite evidenciar la salida o producto final del cuidado de enfermería. 
Los elementos del modelo planteados hasta aquí favorecen el aprendizaje autorregulado y reflexivo, proporcionando estrategias a educadores y estudiantes en la articulación del proceso de enseñanza-aprendizaje. El conocimiento cognitivo se obtiene mediante el uso de estrategias de pensamiento crítico para la comprensión de los diagnósticos de enfermería, sus contenidos, procedimientos y resultados; mientras que el conocimiento metacognitivo se obtiene mediante la reflexión y la auto-regulación para controlar los procesos cognitivos $^{12}$.

El actual modelo orientador del programa de enfermería de la Universidad Industrial de Santander (UIS), se ha fundamentado en la aplicación del proceso de enfermería con el enfoque de la segunda generación, se resalta en este modelo el cuidado desde el nivel individual, familiar o comunitario y el contexto biológico psicosocial, espiritual y ambiental ${ }^{13}$. Sin embargo, es necesario revisarlo con el fin de realizar los ajustes pertinentes, que faciliten la inclusión de aspectos tales como el razonamiento clínico, el mejoramiento de los procesos de aprendizaje, el pensamiento reflexivo, la importancia de la narrativa de los sujetos de cuidado, la integración de un marco conceptual y disciplinar; que favorezca la toma de decisiones y la evaluación de los resultados de enfermería acorde a los nuevos lineamientos estructurados desde el modelo OPT, superando la visión lineal, fraccionada e instrumental de las cinco etapas del proceso de enfermería.

Por lo expuesto anteriormente, este estudio planteó como objetivo general, comparar dos modelos de aplicación del proceso de enfermería (segunda vs tercera generación), en la elaboración de planes de cuidados por parte de estudiantes del programa de enfermería de una institución de educación superior de Bucaramanga (Colombia).

\section{Metodología}

Estudio de corte transversal. Participaron 33 estudiantes de quinto nivel de enfermería UIS, durante el primer semestre de 2018. Las variables en escala de medición cualitativa fueron descritas como frecuencias absolutas y relativas. Las variables continuas se describieron con medidas de tendencia central y de dispersión según sus características.

Inicialmente, con la participación de un experto internacional, se realizó la revisión conceptual del modelo OPT, el estado del arte y experiencias de otras universidades con la aplicabilidad del mismo y se contrastó con el modelo que actualmente tiene el programa de enfermería UIS. Con base en lo anterior y junto con otro experto internacional miembro de la NANDA, se definieron las variables, se diseñaron los instrumentos que permitieron evaluar y comparar la segunda y tercera generación, se diseñó la estrategia pedagógica dirigida a los estudiantes que participaron en la investigación y además se construyeron dos casos (caso 1 y caso 2) de análisis, acordes al nivel académico de los estudiantes.

La estrategia pedagógica se desarrolló durante 10 horas, distribuidas en cuatro momentos:

En el primer momento se entregó a los estudiantes el caso 1 para que lo desarrollaran con base en el modelo segunda generación. Los estudiantes propusieron el plan de cuidados empleando el lenguaje estandarizado NANDA-I, NIC, NOC y el formato SIPCE (sistema de información de las prácticas clínicas y comunitarias) propio del registro de prácticas de estudiantes de enfermería de la UIS y se tuvo acceso al NNN consult (2 horas).

En el segundo momento la experta de NANDA-I presentó a los estudiantes el marco teórico y metodológico del modelo OPT y posteriormente empleó el caso 1 para demostrar la aplicación del modelo (3 horas). En el tercer momento se entregó a los estudiantes el caso 2 para que lo desarrollaran con base en el modelo tercera generación, se les facilitó la plantilla del modelo OPT y la plantilla de la red de razonamiento $1 \mathrm{y}$ se tuvo acceso al NNN Consult (3 horas). En el cuarto momento se aplicó el instrumento de evaluación comparativa y se realizó el análisis y evaluación de los dos modelos segunda y tercera generación (2 horas).

\section{Resultados}

De los 33 estudiantes, el 75,76\% $(n=25)$ eran mujeres y el $24,24 \%(n=8)$ eran hombres. La edad promedio fue 20,5 años IC95\% (20,1 - 21,0).

Como lo muestran la Tabla 1 y la Figura 1, en cuanto a la opinión de los estudiantes sobre la aplicación del proceso de enfermería según ambos modelos, se identificó preferencia por el modelo tercera generación como guía para el abordaje de casos clínicos; sin embargo, en aspectos relacionados con el uso del lenguaje estandarizado, según ítems de comparación del 5 al 8, la mayoría percibió igual aporte por parte de ambos modelos. Especial 
atención tienen los ítems 1,12 y 15, donde cerca del $50 \%$ percibió igualdad entre los modelos y $12 \%$ mencionó el modelo 2 como el de mayor fortaleza. Sin embargo, es importante resaltar que los estudiantes no encontraron diferencias en aspectos de articulación teórica $66.67 \%$, ni en la aplicación de los lenguajes NIC $66.67 \%$ y NOC $57.58 \%$.
Por otra parte, en la comparación de modelos, los estudiantes encontraron diferencias en el uso del modelo OPT en aspectos relacionados con: razonamiento clínico $84.85 \%$, determinación de la esencia del caso $75.76 \%$, respaldo diagnóstico $72.73 \%$ e identificación del diagnóstico de enfermería $66.67 \%$.

Tabla 1. Frecuencia absoluta y relativa de las respuestas al instrumento 6 (comparación modelo segunda o tercera generación $(\mathrm{OPT})) \mathrm{n}=33$ estudiantes.

\begin{tabular}{lcccccc} 
& \multicolumn{2}{c}{ Segunda } & \multicolumn{2}{c}{ Tercera (OPT) } & \multicolumn{2}{c}{ Igual } \\
\cline { 2 - 7 } & $\mathbf{n}$ & $\mathbf{\%}$ & $\mathbf{n}$ & $\mathbf{\%}$ & $\mathbf{n}$ & $\mathbf{\%}$ \\
\hline 1. Datos precisos & 4 & 12,12 & 13 & 39,39 & 16 & 48,48 \\
2. Esencia caso & 2 & 6,06 & 25 & 75,76 & 6 & 18,18 \\
3. Respaldo diag. & 2 & 6,06 & 24 & 72,73 & 7 & 21,21 \\
4. Identificar diag. & 2 & 6,06 & 22 & 66,67 & 9 & 27,27 \\
5. Articulación modelo/teoría & 3 & 9,09 & 8 & 24,24 & 22 & 66,67 \\
6. Estado actual & 2 & 6,06 & 14 & 42,42 & 17 & 51,52 \\
7. NOC & 2 & 6,06 & 12 & 36,36 & 19 & 57,58 \\
8. NIC & 0 & 0 & 11 & 33,33 & 22 & 66,67 \\
11. Juicios clínicos & 0 & 0 & 20 & 60,61 & 13 & 39,39 \\
12. Contexto persona & 4 & 12,12 & 14 & 42,42 & 15 & 45,45 \\
14. Toma de decisiones & 0 & 0 & 20 & 60,61 & 13 & 39,39 \\
15. Experiencia pasada & 4 & 12,12 & 14 & 42,42 & 15 & 45,45 \\
16. Razonamiento clínico & 1 & 3,03 & 28 & 84,85 & 4 & 12,12 \\
\hline
\end{tabular}

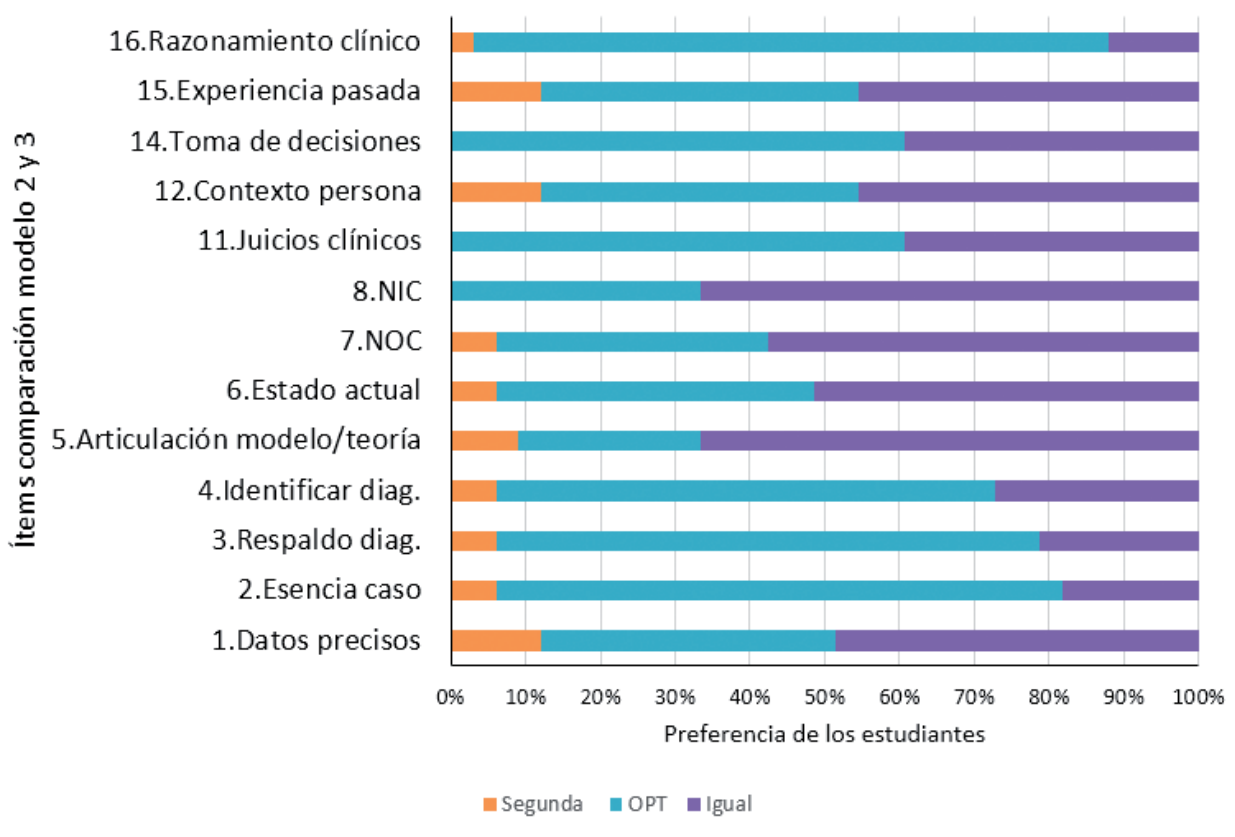

Figura 1. Respuesta opinión estudiantes sobre la aplicación del proceso de enfermería según modelo segunda o tercera generación. 


\section{Discusión}

Las Investigaciones desde el punto de vista educativo, critican el estado actual de aplicación del proceso de enfermería, resaltando aspectos como: poco énfasis en el conocimiento del historial del paciente, la situación del paciente no se analiza dentro de un contexto significativo, es una práctica que no se basa en el pensamiento reflexivo, creativo y no considera los resultados deseados del paciente, haciendo de este un enfoque lineal, escalonado, mecánico, poco reflexivo que limita el desarrollo práctico de reflexiones teóricas relevantes y los procesos meta cognitivos necesarios en el aprendizaje y en el desarrollo de competencias argumentativas y propositivas ${ }^{1}$.

Varios estudios coinciden en afirmar que el modelo OPT posee alta aceptabilidad por los estudiantes en términos de facilitar razonamiento clínico, esencia del caso, respaldo diagnóstico, trabajo en equipo, apoya el aprendizaje autorregulado, refuerza el discernimiento, la toma de decisiones clínicas y se centra en el logro de resultados de calidad ${ }^{8,14-17}$.

En la práctica de cuidado, el proceso de pensamiento conduce a que se produzca razonamiento en todas las fases del proceso de enfermería, asumiendo la responsabilidad para interpretar con precisión las respuestas humanas, con el fin de seleccionar la adecuada evaluación de intervenciones y resultados logrados ${ }^{18,19}$.

El alumno realiza aprehensión de señales y toma decisiones, requiere utilizar el pensamiento crítico para emitir un juicio. Se trata de un conjunto de habilidades previamente aprendidas y actitudes necesarias para el desarrollo del razonamiento clínico ${ }^{18}$.

Pesut y Herman $^{1}$ resaltan la importancia de una representación gráfica de las relaciones funcionales entre hipótesis diagnósticas derivadas de pensamiento sintético para la identificación de temas centrales que requieren el cuidado. La red promueve el razonamiento clínico para identificar y representar sus problemas y ver cómo están relacionados entre sí.

La cuestión clave (cue logic) como elemento de soporte central de la historia de la persona, guía el razonamiento y la atención de planificación basado en un análisis y síntesis de posibilidades diagnósticas como se representa en un razonamiento clínico ${ }^{1}$ y las posibilidades diagnósticas se eligen desde la lista de diagnósticos de enfermería NANDA-I. Por lo tanto, la cuestión clave es el diagnóstico de enfermería primario que organiza el foco diagnóstico, representado en la web de razonamiento clínico. La solución del problema espera que ayude a resolver todos los demás diagnósticos de enfermería relacionados ${ }^{1}$. Lo anterior señala que se debe fortalecer en los estudiantes las mediciones de los indicadores del NOC, su coherencia con el diagnóstico de enfermería y el uso de un modelo o teoría de enfermería ${ }^{1}$.

Una enfermera con experiencia tiene la capacidad de prestar atención a las manifestaciones clínicas que indican una crisis; lo contrario, una incapacidad para ver la prioridad, puede ser lo que precipita un fracaso $^{20}$. La toma de decisiones implica el proceso de selección de las intervenciones de enfermería para ayudar al paciente a alcanzar el estado de resultado deseado $^{1}$. En la práctica clínica es un aspecto que debe ser potencializado para el logro de los resultados propuestos en el plan de cuidados.

El estudio de Bland, et al. ${ }^{20}$ analizó los datos pretest y post-test de encuadre, para determinar si los estudiantes identifican al menos un factor clave en un caso de enfermería psiquiátrica con el uso del modelo OPT. Así, se identificó que se logra una mejoría en la capacidad para enmarcar la historia del paciente y el razonamiento clínico.

La evaluación de problemas del paciente mediante el análisis de datos para identificar problemas dentro del entorno clínico, refleja capacidad de razonamiento clínico de los estudiantes ${ }^{21}$. La aplicación del modelo OPT como estrategia para la enseñanza de razonamiento clínico, mostró mejoras significativas en la capacidad de los estudiantes para enmarcar la historia del paciente, identificar el problema clave de manera correcta, reflexionar sobre sus experiencias y poner sus pensamientos en palabras ${ }^{20,22}$.

En los procesos de formación, se fortalece el desarrollo de competencias genéricas, el razonamiento crítico como potenciador de la autonomía y el aprendizaje a lo largo de toda la vida; así como el desarrollo del hábito de reflexión sobre la práctica diaria, para favorecer toma de decisiones y resolución de problemas propios de la práctica profesional ${ }^{23-25}$.

\section{Conclusiones}

Ampliar la enseñanza del modelo OPT en todos los cursos de enfermería, facilitará una mayor comprensión de los procesos de pensamiento del estudiante y mayor capacidad de adaptar experiencias para satisfacer las necesidades individuales de aprendizaje. 
Se espera que al aplicar el modelo OPT, éste permita mejorar procesos del currículo, procesos pedagógicos y evaluativos; fortalecer buenas prácticas docentes y competencias cognitivas y procedimentales de los estudiantes.

\section{Agradecimientos}

A la Vicerrectoría de Investigación y Extensión de la Universidad Industrial de Santander; a los estudiantes de enfermería que participaron en el estudio y a los expertos internacionales Carmen Espinosa Fresnedo y Howard Butcher.

\section{Consideraciones éticas}

El estudio se realizó de acuerdo a la Resolución No. 08430 de 1993 del Ministerio de Salud y contó con el aval ético del CEINCI UIS (Comité de Ética en Investigación Científica). Se considera una investigación sin riesgo. Los estudiantes firmaron el consentimiento informado y se respetó la confidencialidad de los datos.

\section{Conflictos de interés}

Las autoras declaran no tener conflictos de interés.

\section{Referencias}

1. Pesut DJ, Herman J. OPT: Transformation of nursing process for contemporary practice. Nurs Outlook. 1998; 46(1): 29-36. doi: https://doi.org/10.1016/ s0029-6554(98)90022-7.

2. Pesut DJ. 21ts century nursing knowledge work: reasoning into the future. In Weaver C, Delaney CW, Wewber P, Carr R. (1st ed.) Nursing and informatics for the 21st century: an internacional look at practice, trends and the future. 2006. pp.1323. Chicago IL: HIMSS.

3. Herman H. Clasificación completa de diagnósticos enfermeros NANDA-I 2015-2017. https://www. salusplay.com/blog/diagnosticos-enfermerosnanda-2017/.

4. Bulecheck G, Butcher HK, Dochterman J. Nursing Interventions Classification (NIC) - 5th Ed. 2013. https://www.elsevier.com/ books/nursing-interventions-classification-nic/ bulechek/978-0-323-05340-2.

5. Moorhead Sue, Jhonsson M, Maas M, Swansson E. Nursing Outcomes Classification (NOC) 5th Edition. 2012. https://www.elsevier.com/ books/nursing-outcomes-classification-noc/ moorhead/978-0-323-10010-6.
6. Bartlett R, Bland A, Rossen E, Kautz D, Benfield $\mathrm{S}$, Carnevale T. Evaluation of the outcomepresent state test model as a way to teach clinical reasoning. 2008; 47(8): 337-344. doi: https://doi. org/10.3928/01484834-20080801-01.

7. da Costa CPV, Luz MHBA. Digital learning object for diagnostic reasoning in nursing applied to the integumentary system. Rev Gaucha Enferm. 2015; 36(4): 55-62. doi: https://doi.org/10.1590/19831447.2015.04.54128.

8. Kautz DD, Kuiper R, Pesut DJ, Williams RL. Using NANDA, NIC, and NOC (NNN) language for clinical reasoning with the Outcome-Present StateTest (OPT) model. Int J Nurs Terminol Classif. 2006;17(3): 129-138. doi: https://doi.org/10.1111/ j.1744-618X.2006.00033.x.

9. Pink DH. A whole new mind: moving from the information age to the conceptual age. New York: Riverhead Books; 2005. p282 . http://archive.org/ details/wholenewmindmovi00pink.

10. Herdman H, Kamitsuru S. NANDA International Nursing Diagnoses: definitions and classification 2015 - 2017; 2015. https://www.wiley.com//legacy/ wileychi/nanda/?type=Home.

11. Cowen PS, Moorhead S. Current issues in nursing. St. Louis Mo. Mosby Elsevier; 2011.

12. Kautz D, Kuiper RA, Pesut DJ, Knight-Brown P. Promoting clinical reasoning in undergraduate nursing students: application and evaluation of the outcome present state test (OPT) model of clinical reasoning. Int J Nurs Educ Scholarsh. 2005; 2(1): 1548-923X doi: https://doi.org/10.2202/1548923X.1052.

13. Ibáñez LE, Amaya de Gamarra IC, Niño Bautista L. El proceso de rediseño curricular en el Programa de enfermería de la UIS. Una propuesta Innovadora. Salud UIS. 2002; 34(2): 128-137.

14. Alfayoumi I. The impact of combining conceptbased learning and concept-mapping pedagogies on nursing students' clinical reasoning abilities. Nurse Educ Today. 2019; 72: 40-46. doi: https://doi. org/10.1016/j.nedt.2018.10.009.

15. Jael SA. Use of Outcome-Present state test model of clinical reasoning with Filipino nursing students. Loma Linda University Elect Theses, Dissertations \& Projects. 387. https://scholarsrepository.llu.edu/etd/387.

16. Kaddoura M, Van-Dyke O, Yang Q. Impact of a concept map teaching approach on nursing students' critical thinking skills. Nurs Health Sci. 2016; 18(3): 350-354. doi: https://doi.org/10.1111/ nhs. 12277 . 
17. Griggs K, Arms T. Outcome-present state test model for expanding students' clinical reasoning. Nurse Educator. 2019; 44(3). doi: 10.1097/ NNE.0000000000000578.

18. Carvalho EC, de Souza Oliveira-Kumakura AR, Coelho Ramalho Vasconcelos Morais S. Clinical reasoning in nursing: teaching strategies and assessment tools. 2017; 70(3): 662-668. doi: https:// doi.org/10.1590/0034-7167-2016-0509.

19. Tampy H, Willert E, Ramani S. Assessing Clinical Reasoning: targeting the higher levels of the pyramid. J Gen Intern Med. 2019; 34(8): 1631-1636. doi: https://doi.org/10.1007/s11606-019-04953-4.

20. Bland AR, Rossen EK, Bartlett R, Kautz DD, Carnevale T, Benfield S. Implementation and testing of the OPT MODEL as a teaching strategy in an undergraduate psychiatric nursing course. 2009; 30(1): 14-21.

21. Murphy JI. Using focused reflection and articulation to promote clinical reasoning: An evidence-based teaching strategy. Nurs Educ Perspect. 2004; 25(5): 226-231.

22. Kuiper RA. Integration of innovative clinical reasoning pedagogies into a baccalaureate nursing curriculum. Creat Nurs. 2013; 19(3): 128-139. doi: https://doi.org/10.1891/1078-4535.19.3.128.

23. Roca Llobet J. El Desarrollo del pensamiento crítico a través de diferentes metodologías docentes en el Grado en Enfermería. Universitat Autònoma de Barcelona; 2014. http://hdl.handle. net/10803/129382.

24. Simmons B. Clinical reasoning: concept analysis. J Adv Nurs. 2010; 66(5): 1151-1158. doi: https://doi. org/10.1111/j.1365-2648.2010.05262.x.

25. Rifà Ros R, Pérez Pérez I. Application of an OPT model in a paediatric nursing clinical case in primary health care. Enferm Clin. 2011; 21(3): 168-172. doi: https://doi.org/10.1016/j.enfcli.2010.10.013. 